\title{
Are three-dimensional patient-specific cutting guides for open wedge high tibial osteotomy accurate? An in vitro study
}

\author{
Mathias Donnez ${ }^{1,2,3^{*}}$ (D), Matthieu Ollivier ${ }^{1,2}$, Maxime Munier ${ }^{2}$, Philippe Berton ${ }^{3}$, Jean-Pierre Podgorski ${ }^{3}$, \\ Patrick Chabrand ${ }^{1,2}$ and Sébastien Parratte ${ }^{1,2}$
}

\begin{abstract}
Background: The aim of this in vitro study was to assess the accuracy of three-dimensional patient-specific cutting guides for open wedge high tibial osteotomy (OWHTO) to provide the planned correction in both frontal and sagittal planes.

Methods: Ten cadaveric tibias underwent OWHTO performed using a patient-specific cutting guide based on 3D preoperative planning. An initial CT scan of the tibias was performed, and after segmentation, 3D geometrical models of the pre-OWHTO tibias were obtained. Reference planes were defined, and OWHTO virtually planned to then design patient-specific cutting guides. OWHTO were performed using the patient-specific cutting guides. The patient-specific cutting guide controls the cut and the correction of the OWHTO in both planes. 3D models of post-OWHTO tibias were created after a postoperative CT scan. Geometrical post-OWHTO 3D models were superimposed on pre-OWHTO 3D models. Mechanical medial proximal tibial angle (mMPTA) in the frontal plane and posterior tibial slope (PTS) in the sagittal plane were compared between planned-OWHTO and post-OWHTO 3D reconstructions relative to the preOWHTO reference planes and axis. Pearson's and Lin's correlation tests were performed to assess precision and accuracy of patient-specific cutting guides.
\end{abstract}

Results: The mean difference between post-OWHTO and planned-OWHTO was $0.2^{\circ}$ ( $\max 0.5^{\circ}, \mathrm{SD} 0.3^{\circ}$ ) in the frontal plane and $-0.1^{\circ}\left(\max 0.8^{\circ}, \mathrm{SD} 0.5^{\circ}\right)$ in the sagittal plane. Statistically significant correlations were found between the planned-OWHTO and post-OWHTO configurations for the MMPTA $(p<0.0001)$ and PTS $(p<0.0001)$ measurements, and the bias correction factor was 0.99 in both planes.

Conclusions: 3D patient-specific cutting guides for OWHTO-based 3D virtual planning is a reliable and accurate method of achieving multiplanar correction in both frontal and sagittal planes.

Keywords: Knee surgery, Osteoarthrosis, Medial gonarthrosis, Osteotomy, Open wedge high tibial osteotomy, Patientspecific, Accuracy, Tibial slope correction

\section{Background}

Open wedge high tibial osteotomy (OWHTO) has been described as an efficient conservative surgical treatment preserving the bone stock for patients with moderate medial gonarthrosis and lower leg malalignment $[1,2]$.

\footnotetext{
* Correspondence: mathias.donnez@gmail.com

${ }^{1}$ Aix Marseille Univ, CNRS, ISM, Marseille, France

${ }^{2}$ Aix Marseille Univ, APHM, CNRS, ISM, Sainte-Marguerite Hospital, Institute for Locomotion, Department of Orthopaedics and Traumatology, Marseille, France

Full list of author information is available at the end of the article
}

The objective of the OWHTO is to correct lower leg malalignment in both the frontal and sagittal tibial planes to limit the overload of the medial compartment. Accurate correction is essential to its success as under correction leads to persistent pain and over correction to functional limitations [3, 4].

Different methods and instrumentations have been developed to help the surgeon to achieve the planned correction $[3,5-7]$.

Conventional methods use standard instrumentation to open the osteotomy $[5,6]$. Frontal correction can then 
be managed intraoperatively by measuring the opening angle or opening gap and comparing it to the preoperative plan [8-10], or by using a radiopaque cable under fluoroscopy to control lower limb alignment $[9,11-13]$. The control of the correction for both the frontal and the sagittal planes at the same time with standard instrumentations remains challenging [14-16]. Computer-assisted surgery (CAS) for OWHTO was validated experimentally in vitro by Hankemeier et al. [7] and subsequently used in clinical studies [3, 17-21]. CAS allows real-time control of the correction. All these studies reported better accuracy and reliability for CAS than for conventional or cable methods, but with increased surgical time and a control of the global alignment of the limb but not of tibia only and not the posterior tibial slope (PTS).

While frontal plane correction is most often described for osteotomy management, PTS management in the sagittal plane is essential to preserve biomechanics [22-24]. Song et al. [19] reported that PTS is unchanged if the anterior opening is equal to $67 \%$ of the medial opening. However, this evaluation is complex to perform during surgery, and patient-specific instruments may help the surgeon to manage both the sagittal and the frontal plane correction during surgery $[25,26]$.

Using an experimental setup, three-dimensional patientspecific cutting guides were reported to be more accurate than free-hand technique to perform an osteotomy cut and drill in a synthetic bone [27]. The use of patient-specific cutting guides for OWHTO was also reported recently in three studies realized on small series of patients $[25,26,28]$. All three clinical studies reported good accuracy and reliability of the procedure. Planning procedures for OWHTO were carried out either on 2D long-leg radiographs [28] or from a 3D model [25, 26]. Pérez-Mañanes et al. used CT images to obtain the 3D tibial surface and to position two K-wires to lead the cut, and the correction was planned using two additional wedges.

To avoid the disappointing clinical results observed with patient-specific instrumentation for total knee arthroplasty, we wanted to evaluate in vitro this new technique. After developing a specific patient-specific guide to control both the cut and the correction adapted for a specific OWHTO plate, it was our aim to evaluate the accuracy of the system in an in vitro CT scan-controlled study. Our hypothesis was that the patient-specific cutting guide for OWHTO can provide an accurate correction in both the frontal and sagittal planes.

\section{Methods}

\section{Study design}

In this in vitro study, ten frozen cadaveric specimens' tibias (eight females and two males aged from 70 to 99 , average age 88, 5 right sides) were obtained from our Department of Anatomy at the Aix-Marseille University
School of Medicine (Table 1). The subjects were all preserved in Winckler liquid [29, 30]. All soft tissues were removed, except the patellar tendon insertion.

\section{OWHTO preoperative planning}

All specimens were scanned using a standardized CT scan protocol (Discovery 710, GE Medical System, CERIMED, Marseille, France). The following acquisition parameters were used both prior and after the HTO: $120 \mathrm{kV}, 400 \mathrm{~mA}$, and $0.625-\mathrm{mm}$-thick slices. DICOM images were imported into Mimics 17.0 software (Materialise ${ }^{\circ}$, Leuven, Belgium), and 3D geometrical models of the tibias were created (Pre-OWHTO configurations, Fig. 1).

Anatomical landmarks, anatomical reference planes, and the mechanical axis of the tibia were defined on the 3D models according to Lee et al. [31]. Preoperative mechanical medial proximal tibial angle (mMPTA) and medial PTS were measured (Fig. 2). MMPTA measures the varus deformation of the tibia. PTS measures the sagittal orientation of the proximal tibia. For each specimen, a correction for proximal tibial bony deformity, when present, was determined in both the frontal and the sagittal planes. For specimens with optimal tibial mechanical alignment, a random correction was applied. Each preoperative 3D tibia model was imported to a specially designed 3D planning tool for OWHTO. A cutting plane was positioned, and OWHTO was simulated with respect to the frontal and sagittal plane corrections previously determined (planned-OWHTO configurations, Fig. 1). The Activmotion-2 plate (Newclip Technics ${ }^{\circ}$, Haute-Goulaine, France) was positioned on the anteromedial surface of the tibia following the manufacturer's recommendations. The plate contains four locking screws above and four locking screws below the osteotomy cut. Then, a patient-specific cutting guide was designed for each tibia based on the OWHTO simulation. Patient-specific cutting

Table 1 Specimen description

\begin{tabular}{llllll}
\hline Specimen & Gender & Age & Side & $\begin{array}{l}\text { Pre-OWHTO } \\
\text { mMPTA }\left(^{\circ}\right)\end{array}$ & $\begin{array}{l}\text { Pre-OWHTO } \\
\text { PTS }\left(^{\circ}\right)\end{array}$ \\
\hline G068 & F & 99 & $\mathrm{R}$ & 89.6 & 7.9 \\
G131 & F & 78 & $\mathrm{R}$ & 88.5 & 2.1 \\
G059 & $\mathrm{F}$ & 95 & $\mathrm{~L}$ & 84.2 & 5.3 \\
G141 & $\mathrm{F}$ & 99 & $\mathrm{~L}$ & 88.0 & 2.4 \\
G115 & $\mathrm{M}$ & 88 & $\mathrm{R}$ & 86.9 & 4.7 \\
G111 & $\mathrm{F}$ & 89 & $\mathrm{R}$ & 88.5 & 2.9 \\
G119 & $\mathrm{M}$ & 90 & $\mathrm{R}$ & 88.1 & 4.5 \\
G113 & $\mathrm{F}$ & 84 & $\mathrm{~L}$ & 89.5 & 7.6 \\
G136 & $\mathrm{F}$ & 90 & $\mathrm{~L}$ & 90.3 & 4.2 \\
G117 & $\mathrm{F}$ & 70 & $\mathrm{~L}$ & 83.6 & 9.8 \\
\hline
\end{tabular}

Demographic data, pre-OWHTO measurements 


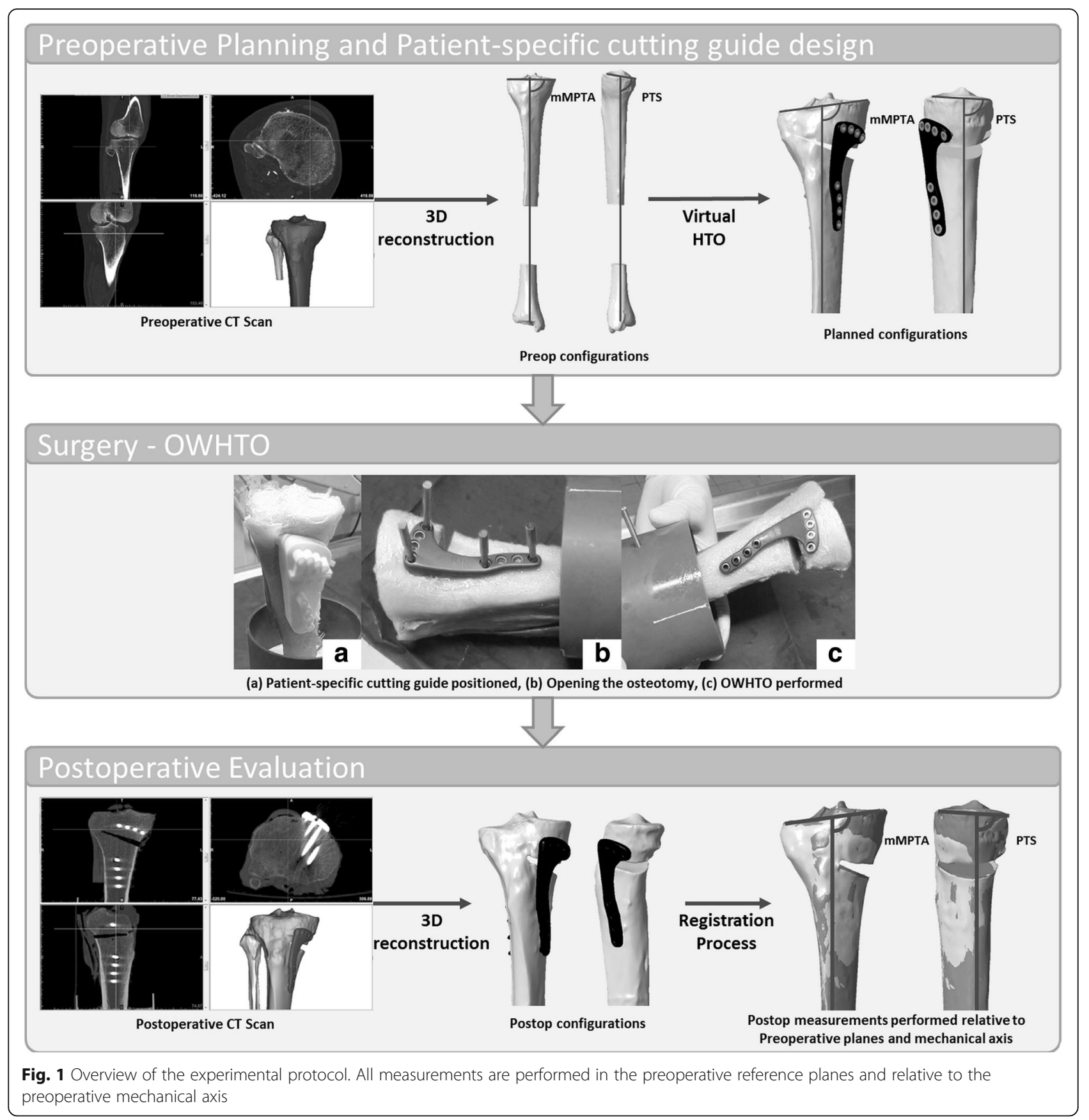

guides took into account the tibial anatomy, the position of the cutting plane, the amount of correction planned in all planes, and the plate location. All patient-specific cutting guides were $3 \mathrm{D}$ printed.

\section{OWHTO}

Specimens were thawed overnight. All OWHTO were performed by one surgeon (MM) using the patient-specific cutting guides [26]. Then, the same CT scan protocol was used to assess the tibias after OWHTO. Post-OWHTO 3D geometrical models were created with Mimics 17.0 software (post-OWHTO configurations, Fig. 1).

\section{Registration process}

For each specimen, the distal part of the post-OWHTO tibia was superimposed on the distal part of the pre-OWHTO tibia using an iterative closest point (ICP) algorithm (Fig. 3). This registration process ensured that measurement references for all models of each tibia were the same. Then, mMPTA and PTS were measured on both the planned-OWHTO and the post-OWHTO 


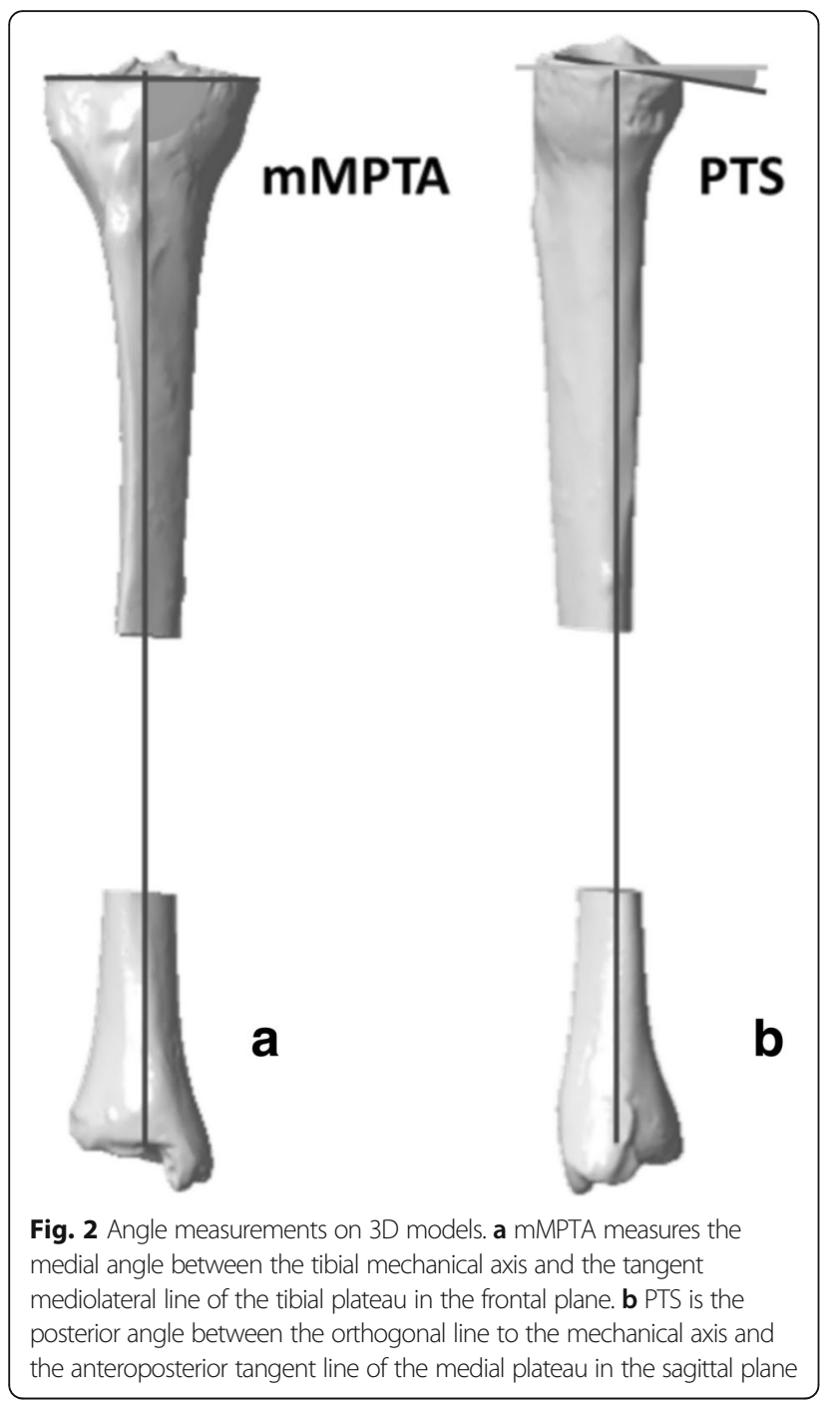

configuration. For maximum reproducibility of measurements, both planned and postoperative angles were measured in the frontal and sagittal pre-OWHTO planes relative to the pre-OWHTO tibial mechanical axis.

Planned-OWHTO and post-OWHTO configurations were compared to assess the precision and the accuracy provided by the patient-specific cutting guides for OWHTO.

\section{Statistical analysis}

Statistical analysis was performed between the plannedOWHTO and the post-OWHTO MMPTA, and between the planned-OWHTO and the post-OWHTO PTS. Correlation analyses were performed using Pearson's correlation test to assess the precision reached by the patient-specific cutting guides. Accuracy was assessed by the calculation of the bias correction factor $\left(C_{\mathrm{b}}\right)$ given by Lin's correlation test [32].

Significance was considered at $p<0.05$. The $95 \%$ confidence intervals $(\mathrm{CI})$ were presented. Statistical analysis was performed on R software.

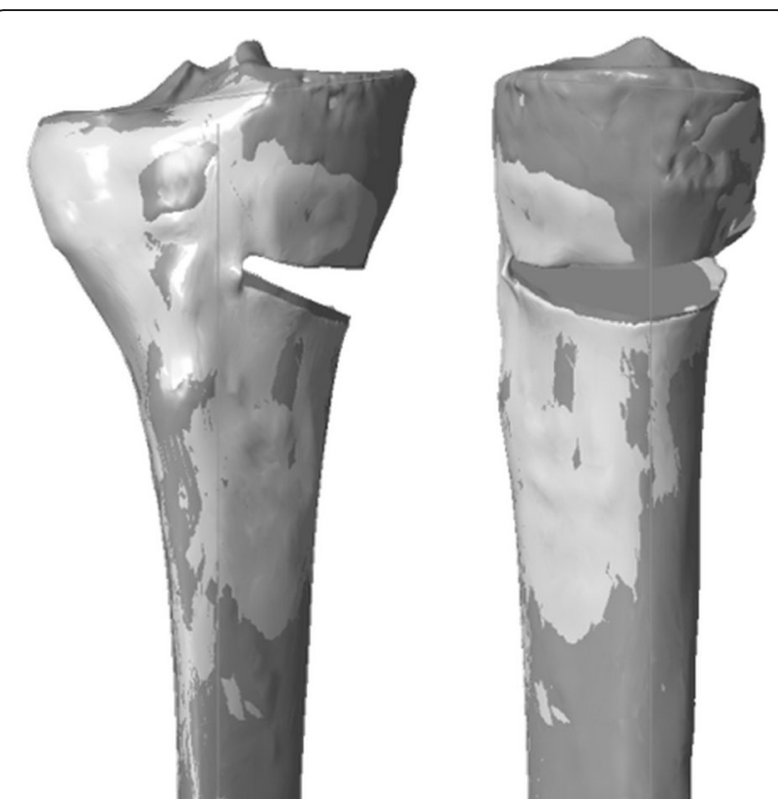

Fig. 3 The post-OWHTO model was registered on the planned-OWHTO mode

\section{Results}

The mean pre-OWHTO mMPTA was $87.7^{\circ}$ (SD $2.2^{\circ}$ ), and the mean pre-OWHTO PTS was $5.1^{\circ}$ (SD 2.6 ). A mean $7.3^{\circ}\left(\mathrm{SD} 1.3^{\circ}\right)$ correction in the frontal plane and a $3.3^{\circ}\left(\mathrm{SD} 2.7^{\circ}\right)$ correction in the sagittal plane were thus planned. For all specimens, the positioning of the patient-specific cutting guides on the bone was possible in accordance with the planning. All patient-specific cutting guides fitted the tibial surface, so all OWHTO were performed as planned.

Differences between the post-OWHTO and plannedOWHTO configurations were calculated. The mean difference between the post-OWHTO and plannedOWHTO configurations was $0.2^{\circ}$ (from $-0.3^{\circ}$ to $0.5^{\circ}, \mathrm{SD}$ $0.3^{\circ}$ ) in the frontal plane, and $-0.1^{\circ}$ (from $-0.7^{\circ}$ to $0.8^{\circ}$, $\mathrm{SD} 0.5^{\circ}$ ) in the sagittal plane (Table 2).

According to Pearson's correlation tests, a statistically significant correlation was found between the planned-OWHTO and post-OWHTO configurations, for the mMPTA measurements with a correlation coefficient of 0.99 (95\% CI, 94-99\%, $p<0.0001$ ), and for the PTS measurements with a correlation coefficient of 0.97 (95\% CI, 86-99\%, $p<0.0001$ ) (Fig. 4).

Bias coefficient $C_{\mathrm{b}}$ was 0.99 in both the frontal and sagittal planes (Table 3).

\section{Discussion}

OWHTO success remains on accurate correction in order to avoid persistent pain or functional limitations [3, 4]. This in vitro study aimed to investigate the accuracy in both the frontal and sagittal planes provided by 
Table 2 Angular measurements

\begin{tabular}{|c|c|c|c|c|c|c|c|c|c|c|}
\hline \multirow[b]{2}{*}{ Specimen } & \multicolumn{2}{|l|}{ Pre-OWHTO } & \multicolumn{2}{|l|}{ Corrections } & \multicolumn{2}{|c|}{ Planned-OWHTO } & \multicolumn{2}{|c|}{ Post-OWHTO } & \multicolumn{2}{|c|}{ Planned-OWHTO vs post-OWHTO } \\
\hline & mMPTA $\left(^{\circ}\right)$ & PTS $\left({ }^{\circ}\right)$ & mMPTA $\left(^{\circ}\right)$ & PTS $\left(^{\circ}\right)$ & mMPTA $\left({ }^{\circ}\right)$ & PTS $\left({ }^{\circ}\right)$ & mMPTA $\left(^{\circ}\right)$ & $\overline{\mathrm{PTS}}\left({ }^{\circ}\right)$ & mMPTA $\left(^{\circ}\right)$ & PTS $\left(^{\circ}\right)$ \\
\hline G068 & 89.6 & 7.9 & 8 & 5 & 97.3 & 3.0 & 97.0 & 3.5 & 0.3 & -0.5 \\
\hline G131 & 88.5 & 2.1 & 6 & 0 & 94.5 & 2.1 & 94.7 & 2.3 & -0.2 & -0.2 \\
\hline G059 & 84.2 & 5.3 & 8 & 0 & 92.9 & 5.2 & 92.9 & 4.4 & 0.0 & 0.8 \\
\hline G141 & 88.0 & 2.4 & 9 & 3 & 97.0 & -0.7 & 96.6 & -0.6 & 0.4 & -0.1 \\
\hline G115 & 86.9 & 4.7 & 8 & 4 & 95.2 & 0.7 & 94.7 & 1.2 & 0.5 & -0.5 \\
\hline G111 & 88.5 & 2.9 & 7 & 3 & 95.1 & -0.1 & 95.2 & 0.3 & -0.1 & -0.4 \\
\hline G119 & 88.1 & 4.5 & 6 & 4 & 94.1 & 0.6 & 93.6 & 0.9 & 0.5 & -0.3 \\
\hline G113 & 89.5 & 7.6 & 5 & 6 & 94.1 & 1.7 & 93.7 & 1.1 & 0.4 & 0.6 \\
\hline G136 & 90.3 & 4.2 & 7 & 0 & 97.3 & 4.1 & 97.6 & 3.9 & -0.3 & 0.2 \\
\hline G117 & 83.6 & 9.8 & 9 & 8 & 92.2 & 1.9 & 91.8 & 2.6 & 0.4 & -0.7 \\
\hline
\end{tabular}

Pre-OWHTO measurements, planned corrections, planned-OWHTO measurements, post-OWHTO measurements, and absolute difference between planned-OWHTO and post-OWHTO configurations

patient-specific cutting guides with respect to 3D planning of OWHTO. The hypothesis that patient-specific cutting guides can provide the planned correction in both the frontal and sagittal planes was confirmed in this CT scan-controlled in vitro study.

In this patient-specific procedure for OWHTO, bone deformation angles were measured with respect to the anatomical reference planes determined using the procedure of Lee et al. [31] in order to mimic clinical practice. Clinical studies report several methods of finding anatomical reference points and measuring deformation angles [25]. Seeking to achieve maximum reliability and to measure only the correction provided by the patient-specific cutting guide, all measurements were performed relative to the same axis and in the same planes for the pre-OWHTO, planned-OWHTO, and post-OWHTO configurations. Unlike Munier et al., who performed measurements separately on the preoperative and postoperative 3D configurations, and then compared how far the two configurations differed from the planned correction, we performed our measurements relative to the preoperative mechanical axis. This was made possible by the registration process we used to superimpose the post-OWHTO configuration on the
planned-OWHTO configuration. As our objective was to assess the amount of correction, it was vital to keep the reference definition the same for both plannedOWHTO and post-OWHTO configurations.

Intraoperative methods to control correction are varied, have limited accuracy, and mainly focus on the frontal plane correction. In their review of the literature, Van Den Bempt et al. reported ranges of accuracy in the frontal plane from several clinical studies [4]. For conventional methods, the mean amplitude of the range of accuracy was $5.6^{\circ}$ (from $4^{\circ}$ to $8^{\circ}$ ) [33-37], whereas using the CAS method, it was $5.5^{\circ}$ (from $4^{\circ}$ to $7^{\circ}$ ) $[10,17,38-41]$. Irrespective of the range of accuracy chosen by the authors, the literature reports a mean $32 \%$ of outlier patients when authors used conventional methods [9, 10, 17, 21,34-37, 40, 42-45] and 22\% when they used CAS $[10,17,21,38-42,44,46]$. Some in vitro studies find CAS to be accurate for OWHTO. On a single synthetic bone and with a statistical model, Keppler et al. [47] evaluated a mean error of $0.7^{\circ}$ in the frontal plane between the postoperative results and the target. Wang et al. [48] performed OWHTO with several amounts of correction in a synthetic bone using CAS and reported $0.4^{\circ}$ accuracy in the frontal plane. Both authors validated their
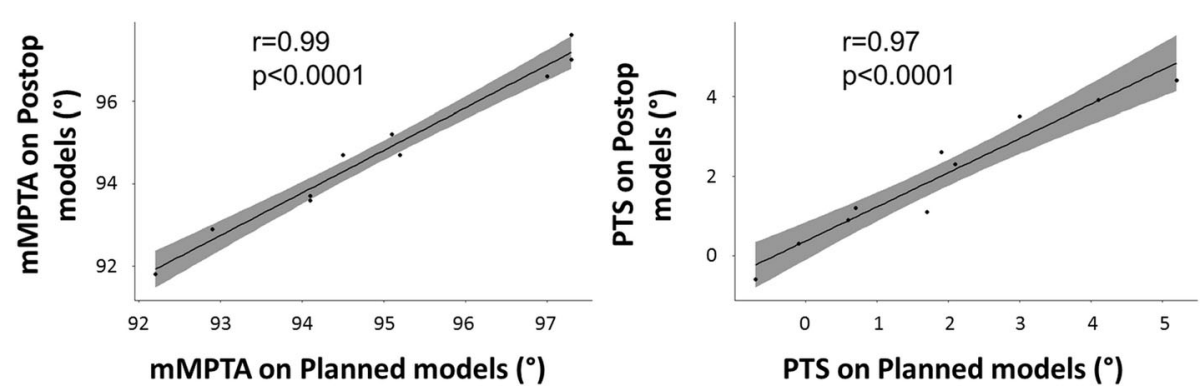

Fig. 4 Correlations between planned-OWHTO and postop-OWHTO models for MMPTA (a) and PTS (b) 
Table 3 Statistical analysis results

\begin{tabular}{lll}
\hline & Pearson's correlation coefficient & $C_{\mathrm{b}}$ \\
\hline mMPTA & $0.99(95 \% \mathrm{Cl}, 94-99 \%)$ & 0.99 \\
PTS & $0.97(95 \% \mathrm{Cl}, 86-99 \%)$ & 0.99
\end{tabular}

Pearson's correlation coefficient and bias correction factor $C_{\mathrm{b}}$ between planned-OWHTO and post-OWHTO configurations

experimental work in a preliminary clinical study, on five and four patients. They both found a mean error of $1^{\circ}$ in the frontal plane. On a single cadaveric specimen, Lützner et al. [49] evaluated the accuracy of CAS to measure lower limb alignment. They found a mean error of $0.6^{\circ}$ in the frontal plane. Hankemeier et al. [7] performed OWHTO on 20 legs randomly assigned to CAS or a conventional method. They reported better accuracy and less variability than with a cable method. Among clinical studies using patient-specific cutting guides, Menetrey et al. [20] evaluated the frontal plane correction, based on 2D measurements for planning. Their patient-specific cutting guide only incorporates the cutting plane position, the correction being guided by two additional wedges. They found $0.5^{\circ}$ accuracy (from $0^{\circ}$ to $1.2^{\circ}$ ) in the frontal plane. Patient-specific cutting guides containing both the cutting plane planning and the correction were used in two other studies [19, 21]. In one, Munier et al. performed a postoperative 3D evaluation of their patients. Overall, they found similar accuracy for both 2D and 3D measurements by reproducing the measurement protocol on the postoperative model [26] around $0.0^{\circ}$ (from $-1.7^{\circ}$ to $1.8^{\circ}, \mathrm{SD} 1.1^{\circ}$ ) in the frontal plane. In the other study, Victor and Premanathan [25] reported a mean difference of $0.1^{\circ}$ (from $-1^{\circ}$ to $1^{\circ}, \mathrm{SD}-0.1^{\circ}$ ) in the frontal plane.

Controlling the sagittal plane correction is essential to preserve knee biomechanics [23-25], but conventional methods have limited accuracy, remaining on gap measurements $[15,19]$. Using CAS on synthetic bones, a mean error of $0.9^{\circ}$ [47] and a $0.5^{\circ}$ accuracy [48] were reported in the sagittal plane. Using patient-specific cutting guides, an accuracy around $0.3^{\circ}$ (from $-2^{\circ}$ to $3.2^{\circ}$, SD $1.4^{\circ}$ ) [26] and a mean difference of $-0.1^{\circ}$ (from $-3^{\circ}$ to $2^{\circ}, \mathrm{SD} 1.2^{\circ}$ ) [25] in the sagittal plane were reported.

In this study, the patient-specific OWHTO procedure includes $3 \mathrm{D}$ preoperative planning of the surgery and the design of a patient-specific cutting guide which takes into account the tibial anatomy, the position of the cutting plane, the amount of correction planned in all planes, and the plate location. The mean differences between the planned-OWHTO and post-OWHTO models are $0.2^{\circ}$ (from $-0.3^{\circ}$ to $0.5^{\circ}, \mathrm{SD} 0.3^{\circ}$ ) in the frontal plane and $-0.1^{\circ}$ (from $-0.7^{\circ}$ to $0.8^{\circ}, \mathrm{SD} 0.5^{\circ}$ ) in the sagittal plane. OWHTO outcomes are compared with the planning by superimposing the post-HTO $3 \mathrm{D}$ reconstruction on the planning 3D model. This enabled to assess the accuracy of patient-specific cutting guides. Findings of the present study suggest that PTS is managed with accuracy, which is important for the management of cruciate ligament balance $[23,25]$.

One limitation of our study is the small number of specimens used. Moreover, not all specimens had a sufficient varus deformation in their proximal tibia to be considered as candidates for OWHTO. However, different degrees of correction consistent with clinical practice were planned in both the frontal and sagittal planes. We also departed from clinical practice in performing all the measurements on 3D models. This was possible thanks to the cadaveric nature of the specimens. This enabled us to assess precision and accuracy by comparing post-OWHTO and planned-OWHTO 3D models, thereby avoiding measurement errors related to $2 \mathrm{D}$ radiography. The preoperative $\mathrm{CT}$ scan required for this procedure could be another limitation for direct clinical application, not being necessary in conventional or CAS procedures. However, Menetrey et al. [20] reported that the patient-specific cutting guide reduced the use of intraoperative fluoroscopy from 55 images on average (range, 41-73) in conventional methods to 8 (range, 6$14)$, as well as requiring less surgical time.

\section{Conclusion}

OWHTO is demanding surgery, and accuracy of the correction in both frontal and sagittal planes is essential to its success. This study shows that combining 3D planning with patient-specific cutting guides for OWHTO is a reliable and accurate method of achieving multiplanar correction in both the frontal and the sagittal planes. Further randomized clinical studies should be carried out to validate these experimental results and evaluate the risk-benefit ratio of the preoperative CT scan, and the reduction in surgical time.

\footnotetext{
Acknowledgements

We would like to thank Pauline Brige (CERIMED, Aix-Marseille University, Marseille, France) for her help in the CT acquisitions. We also thank Marjorie Sweetko for re-reading the English of this manuscript.
}

\section{Availability of data and materials}

The datasets used and/or analyzed during the current study are available from the corresponding author on reasonable request.

\section{Authors' contributions}

MD participated in the conception and design of the study and in the acquisition and interpretation of the data and was a major contributor in writing the manuscript. MO participated in the conception of the study and in the acquisition of the data and was a major contributor in writing the manuscript. MM performed the OWHTO and participated in the acquisition and interpretation of the data. PB and JPP participated in the conception and design of the study. SP and PC were involved in the conception and design of the study and in the critical revision of the manuscript. All authors read and approved the final manuscript.

\section{Ethics approval and consent to participate}

Local ethics committee approval was not required because of the use of cadaveric disidentified specimens. The scientific committee of our anatomical department approved our study protocol. 


\section{Consent for publication}

Not applicable.

\section{Competing interests}

The authors declare that they have no competing interests.

\section{Publisher's Note}

Springer Nature remains neutral with regard to jurisdictional claims in published maps and institutional affiliations.

\section{Author details}

${ }^{1}$ Aix Marseille Univ, CNRS, ISM, Marseille, France. ${ }^{2}$ Aix Marseille Univ, APHM, CNRS, ISM, Sainte-Marguerite Hospital, Institute for Locomotion, Department of Orthopaedics and Traumatology, Marseille, France. ${ }^{3}$ Newclip Technics, Haute-Goulaine, France.

\section{Received: 16 May 2018 Accepted: 25 June 2018}

\section{Published online: 09 July 2018}

\section{References}

1. Flecher X, Parratte S, Aubaniac J-M, J-NA A. A 12-28-year followup study of closing wedge high tibial osteotomy. Clin Orthop. 2006;452:91-6. https:// doi.org/10.1097/01.blo.0000229362.12244.f6.

2. Amendola A, Bonasia DE. Results of high tibial osteotomy: review of the literature. Int Orthop. 2010;34:155-60. https://doi.org/10.1007/s00264-0090889-8.

3. Saragaglia D, Chedal-Bornu B, Rouchy RC, Rubens-Duval B, Mader R, Pailhé $R$. Role of computer-assisted surgery in osteotomies around the knee. Knee Surg Sports Traumatol Arthrosc. 2016;24:3387-95. https://doi.org/10.1007/ s00167-016-4302-z

4. Van den Bempt M, Van Genechten W, Claes T, Claes S. How accurately does high tibial osteotomy correct the mechanical axis of an arthritic varus knee? A systematic review. Knee. 2016;23:925-35. https://doi.org/10.1016/j.knee. 2016.10.001.

5. Lobenhoffer P, Agneskirchner JD. Improvements in surgical technique of valgus high tibial osteotomy. Knee Surg Sports Traumatol Arthrosc. 2003;11: 132-8. https://doi.org/10.1007/s00167-002-0334-7.

6. Staubli AE, Jacob HAC. Evolution of open-wedge high-tibial osteotomy: experience with a special angular stable device for internal fixation without interposition material. Int Orthop. 2010;34:167-72. https://doi.org/10.1007/ s00264-009-0902-2.

7. Hankemeier S, Hufner T, Wang G, Kendoff D, Zeichen J, Zheng G, et al Navigated open-wedge high tibial osteotomy: advantages and disadvantages compared to the conventional technique in a cadaver study. Knee Surg Sports Traumatol Arthrosc. 2006;14:917-21. https://doi.org/10. 1007/s00167-006-0035-8

8. Elson DW, Petheram TG, Dawson MJ. High reliability in digital planning of medial opening wedge high tibial osteotomy, using Miniaci's method. Knee Surg Sports Traumatol Arthrosc. 2015;23:2041-8. https://doi.org/10.1007/ s00167-014-2920-X

9. Yoon S-D, Zhang G, Kim H-J, Lee B-J, Kyung H-S. Comparison of cable method and Miniaci method using picture archiving and communication system in preoperative planning for open wedge high tibial osteotomy. Knee Surg Relat Res. 2016;28:283-8. https://doi.org/10.5792/ksrr.16.052.

10. Schröter S, Ihle C, Elson DW, Döbele S, Stöckle U, Ateschrang A. Surgical accuracy in high tibial osteotomy: coronal equivalence of computer navigation and gap measurement. Knee Surg Sports Traumatol Arthrosc. 2016:24:3410-7. https://doi.org/10.1007/s00167-016-3983-7.

11. Fujisawa $Y$, Masuhara K, Shiomi S. The effect of high tibial osteotomy on osteoarthritis of the knee. An arthroscopic study of 54 knee joints. Orthop Clin North Am. 1979;10:585-608.

12. Dugdale TW, Noyes FR, Styer D. Preoperative planning for high tibial osteotomy. The effect of lateral tibiofemoral separation and tibiofemoral length. Clin Orthop. 1992;274:248-64.

13. Kim MS, Son JM, Koh IJ, Bahk JH, In Y. Intraoperative adjustment of alignment under valgus stress reduces outliers in patients undergoing medial opening-wedge high tibial osteotomy. Arch Orthop Trauma Surg. 2017:1-11. https://doi.org/10.1007/s00402-017-2729-4.

14. Hernigou P. Open wedge tibial osteotomy: combined coronal and sagittal correction. Knee. 2002;9:15-20. https://doi.org/10.1016/S0968-0160(01)00111-9.
15. Noyes FR, Goebel SX, West J. Opening wedge tibial osteotomy: the 3triangle method to correct axial alignment and tibial slope. Am J Sports Med. 2005;33:378-87.

16. Hinterwimmer S, Beitzel K, Paul J, Kirchhoff C, Sauerschnig M, von Eisenhart-Rothe $\mathrm{R}$, et al. Control of posterior tibial slope and patellar height in open-wedge valgus high tibial osteotomy. Am J Sports Med. 2011;39:851-6. https://doi.org/10.1177/0363546510388929.

17. Saragaglia D, Roberts J. Navigated osteotomies around the knee in 170 patients with osteoarthritis secondary to genu varum. Orthopedics. 2005;28:s1269-74.

18. Maurer F, Wassmer G. High tibial osteotomy: does navigation improve results? Orthopedics. 2006;29:S130-2.

19. Song E-K, Seon J-K, Park S-J. How to avoid unintended increase of posterior slope in navigation-assisted open-wedge high tibial osteotomy. Orthopedics. 2007:30:S127-31.

20. Menetrey J, Duthon V, Fritschy D. Computer-assisted open-wedge high tibial osteotomy. Oper Tech Orthop. 2008;18:210-4. https://doi.org/10. 1053/j.oto.2008.12.003

21. Chang J, Scallon G, Beckert M, Zavala J, Bollier M, Wolf B, et al. Comparing the accuracy of high tibial osteotomies between computer navigation and conventional methods. Comput Assist Surg Abingdon Engl. 2017;22(1):-8. https://doi.org/10.1080/24699322.2016.1271909.

22. Rodner CM, Adams DJ, Diaz-Doran V, Tate JP, Santangelo SA, Mazzocca $A D$, et al. Medial opening wedge tibial osteotomy and the sagittal plane. Am J Sports Med. 2006;34:1431-41. https://doi.org/10.1177/ 0363546506287297

23. Martineau PA, Fening SD, Miniaci A. Anterior opening wedge high tibial osteotomy: the effect of increasing posterior tibial slope on ligament strain. Can J Surg. 2010;53:261-7.

24. Akamatsu Y, Sotozawa M, Kobayashi H, Kusayama Y, Kumagai K, Saito T. Usefulness of long tibial axis to measure medial tibial slope for opening wedge high tibial osteotomy. Knee Surg Sports Traumatol Arthrosc. 2014:17. https://doi.org/10.1007/s00167-014-3403-9.

25. Victor J, Premanathan A. Virtual 3D planning and patient specific surgical guides for osteotomies around the knee. Bone Jt J. 2013;95-B:153-8. https:// doi.org/10.1302/0301-620X.95B11.32950.

26. Munier M, Donnez M, Ollivier M, Flecher X, Chabrand P, Argenson J-N, et al. Can three-dimensional patient-specific cutting guides be used to achieve optimal correction for high tibial osteotomy? Pilot study. Orthop Traumatol Surg Res n.d. doi:https://doi.org/10.1016/j.otsr.2016.11.020.

27. Sys G, Eykens H, Lenaerts G, Shumelinsky F, Robbrecht C, Poffyn B. Accuracy assessment of surgical planning and three-dimensional-printed patientspecific guides for orthopaedic osteotomies. Proc Inst Mech Eng [H]. 2017 231:499-508. https://doi.org/10.1177/0954411917702177.

28. Pérez-Mañanes R, Burró J, Manaute J, Rodriguez F, Martín J. 3D surgical printing cutting guides for open-wedge high Tibial osteotomy: do it yourself n.d. doi:https://doi.org/10.1055/s-0036-1572412.

29. Winckler G. Manuel d'anatomie topographique et fonctionnelle. Masson et Cie Niort, impr. Soulisse et Cassegrain. 1964.

30. Crandall J. The preservation of human surrogates for biomechanical studies: University of Virginia; 1994

31. Lee YS, Park SJ, Shin VI, Lee JH, Kim YH, Song EK. Achievement of targeted posterior slope in the medial opening wedge high tibial osteotomy: a mathematical approach. Ann Biomed Eng. 2009;38:583-93. https://doi.org/ 10.1007/s10439-009-9860-5.

32. Lin LI-K. A concordance correlation coefficient to evaluate reproducibility. Biometrics. 1989:45:255-68. https://doi.org/10.2307/2532051.

33. Magyar G, Ahl TL, Vibe P, Toksvig-Larsen S, Lindstrand A. Open-wedge osteotomy by hemicallotasis or the closed-wedge technique for osteoarthritis of the knee. J Bone Jt Surg Br. 1999:81-B:444-8.

34. Brouwer RW, Bierma-Zeinstra SMA, van Raaij TM, Verhaar J a N. Osteotomy for medial compartment arthritis of the knee using a closing wedge or an opening wedge controlled by a Puddu plate. Bone Jt J. 2006;88-B:1454-9. https://doi.org/10.1302/0301-620X.88B11.17743.

35. Hankemeier S, Mommsen P, Krettek C, Jagodzinski M, Brand J, Meyer C, et al. Accuracy of high tibial osteotomy: comparison between open- and closed-wedge technique. Knee Surg Sports Traumatol Arthrosc. 2009;18: 1328-33. https://doi.org/10.1007/s00167-009-1020-9.

36. Brosset T, Pasquier G, Migaud H, Gougeon F. Opening wedge high tibial osteotomy performed without filling the defect but with locking plate fixation (TomoFix ${ }^{\mathrm{TM}}$ ) and early weight-bearing: prospective evaluation of bone 
union, precision and maintenance of correction in 51 cases. Orthop Traumatol Surg Res OTSR. 2011;97:705-11. https:/doi.org/10.1016/.otsr.2011.06.011.

37. Duivenvoorden T, Brouwer RW, Baan A, Bos PK, Reijman M, Bierma-Zeinstra SMA, et al. Comparison of closing-wedge and opening-wedge high tibial osteotomy for medial compartment osteoarthritis of the knee: a randomized controlled trial with a six-year follow-up. J Bone Joint Surg Am. 2014;96:1425-32. https://doi.org/10.2106/JBJS.M.00786.

38. Bae DK, Song SJ, Yoon KH. Closed-wedge high tibial osteotomy using computer-assisted surgery compared to the conventional technique. Bone Jt J. 2009;91-B:1 164-71. https://doi.org/10.1302/0301-620X.91B9.22058.

39. Gebhard F, Krettek C, Hüfner T, Grützner PA, Stöckle U, Imhoff AB, et al. Reliability of computer-assisted surgery as an intraoperative ruler in navigated high tibial osteotomy. Arch Orthop Trauma Surg. 2011;131:297302. https://doi.org/10.1007/s00402-010-1145-9.

40. Iorio R, Pagnottelli M, Vadalà A, Giannetti S, Sette PD, Papandrea P, et al. Open-wedge high tibial osteotomy: comparison between manual and computer-assisted techniques. Knee Surg Sports Traumatol Arthrosc. 2011; 21:113-9. https://doi.org/10.1007/s00167-011-1785-5.

41. Lee D-H, Nha K-W, Park S-J, Han S-B. Preoperative and postoperative comparisons of navigation and radiologic limb alignment measurements after high tibial osteotomy. Arthrosc J Arthrosc Relat Surg. 2012;28:1842-50. https://doi.org/10.1016/j.arthro.2012.05.881.

42. Stanley JC, Robinson KG, Devitt BM, Richmond AK, Webster KE, Whitehead TS, et al. Computer assisted alignment of opening wedge high tibial osteotomy provides limited improvement of radiographic outcomes compared to flouroscopic alignment. Knee. 2016;23:289-94. https://doi.org/ 10.1016/j.knee.2015.12.006.

43. Marti CB, Gautier E, Wachtl SW, Jakob RP. Accuracy of frontal and sagittal plane correction in open-wedge high tibial osteotomy. Arthrosc J Arthrosc Relat Surg. 2004;20:366-72. https://doi.org/10.1016/j.arthro.2004.01.024.

44. Lee D-H, Han S-B, Oh K-J, Lee JS, Kwon J-H, Kim J-I, et al. The weight-bearing scanogram technique provides better coronal limb alignment than the navigation technique in open high tibial osteotomy. Knee. 2014;21:451-5. https:/doi.org/10.1016/j.knee.2012.09.003.

45. El-Azab HM, Morgenstern M, Ahrens P, Schuster T, Imhoff AB, Lorenz SGF. Limb alignment after open-wedge high tibial osteotomy and its effect on the clinical outcome. Orthopedics. 2011;34:e622-8. https://doi.org/10.3928/ 01477447-20110826-02.

46. Lee D-H, Park S-C, Park H-J, Han S-B. Effect of soft tissue laxity of the knee joint on limb alignment correction in open-wedge high tibial osteotomy. Knee Surg Sports Traumatol Arthrosc. 2016;24:3704-12. https://doi.org/10. 1007/s00167-015-3682-9.

47. Keppler P, Gebhard F, Grützner PA, Wang G, Zheng G, Hüfner T, et al. Computer aided high tibial open wedge osteotomy. Injury. 2004;35:68-78. https://doi.org/10.1016/j.injury.2004.05.013.

48. Wang G, Zheng G, Keppler P, Gebhard F, Staubli A, Mueller U, et al. Implementation, accuracy evaluation, and preliminary clinical trial of a CTfree navigation system for high tibial opening wedge osteotomy. Comput Aided Surg Off J Int Soc Comput Aided Surg. 2005;10:73-85. https://doi.org/ 10.3109/10929080500228837.

49. Lützner J, Groß A, Günther K, Kirschner S. Reliability of limb alignment measurement for high tibial osteotomy with a navigation system. Eur J Med Res. 2009;14:447-50. https://doi.org/10.1186/2047-783X-14-10-447.

\section{Ready to submit your research? Choose BMC and benefit from:}

- fast, convenient online submission

- thorough peer review by experienced researchers in your field

- rapid publication on acceptance

- support for research data, including large and complex data types

- gold Open Access which fosters wider collaboration and increased citations

- maximum visibility for your research: over $100 \mathrm{M}$ website views per year

At BMC, research is always in progress.

Learn more biomedcentral.com/submissions 